\title{
Treatment and prognosis for patients with differentiated thyroid carcinoma bone metastases
}

\author{
Shiqi Wang, Juanzhang Luo, Zhaoming Zhong, Xiao Qi, Ting Liu, Rujia Qin, Zaili Zhao, Yan Xi, \\ Ruimei Sun, Yaohong Wu, Chuanzheng Sun \\ Department of Head \& Neck Surgery Section II, Third Affiliated Hospital of Kunming Medical University, Kunming 650118, China \\ Contributions: (I) Conception and design: C Sun, Y Xi, R Sun; (II) Administrative support: Y Wu; (III) Provision of study patients: X Qin, T Liu, R \\ Qin, Z Zhao; (IV) Collection and assembly of data: J Luo, Z Zhong; (V) Data analysis and interpretation: S Wang, C Sun; (VI) Manuscript writing: \\ All authors; (VII) Final approval of manuscript: All authors. \\ Correspondence to: Chuanzheng Sun. Department of Head \& Neck Surgery Section II, Third Affiliated Hospital of Kunming Medical University, \\ Tumor Hospital of Yunnan Province, No. 519 Kunzhou Road, Kunming 650118, China. Email: scz008@126.com.
}

Background: To investigate reasonable treatment modalities and prognostic factors for patients with differentiated thyroid carcinoma (DTC) bone metastases (BM).

Methods: The clinicopathological characteristics and follow-up data for all patients with DTC BM who received treatment in the Third Affiliated Hospital of Kunming Medical University between November 1, 1993 and December 31, 2016 were analyzed retrospectively with respect to mortality and survival rates. Multivariate analysis was performed using the Cox proportional hazard model.

Results: The 5- and 10-year overall survival (OS) rates were 51.5\% and $17.2 \%$, respectively, for all 60 patients. Univariate analysis showed that patients diagnosed with BM at $<45$ years of age, with controlled thyroid-stimulating hormone (TSH) levels and those undergoing surgery or ${ }^{131} \mathrm{I}$ therapy had better prognoses. Patients with cervical vertebra metastases, multiple organ metastases other than bone, and those receiving chemotherapy had worse prognoses. Gender, pathological type, number of BM lesions, skeletalrelated events (SREs) and whether or not the patient received radiotherapy were not related to prognosis. Cox regression analysis showed that age at diagnosis of BM, undergoing surgery for bone lesions, and not receiving chemotherapy were independent factors of favorable prognosis for patients with DTC BM.

Conclusions: Patients with DTC BM have poor prognoses. Age at diagnosis with BM $<45$ years old, undergoing surgery for bone lesions, and not receiving chemotherapy were independent factors of favorable prognosis for patients with DTC BM. ${ }^{131} \mathrm{I}$ combined with surgery for bone metastatic lesions may be the best treatment model for most patients with DTC BM disease.

Keywords: Head and neck cancer; differentiated thyroid carcinoma (DTC); bone metastases (BM); treatment; prognosis

Submitted Sep 19, 2019. Accepted for publication Nov 26, 2019.

doi: $10.21037 /$ tcr.2019.12.43

View this article at: http://dx.doi.org/10.21037/tcr.2019.12.43

\section{Introduction}

Differentiated thyroid carcinoma (DTC) accounts for approximately $85-90 \%$ of all thyroid cancers $(1,2)$. DTC patients usually have good prognosis with a 10 -year survival rate over $85-90 \%$ if appropriate treatment is given (3); however, a few DTC patients will suffer from recurrence and metastatic disease. Bone is the second most-common site of distant metastasis for thyroid cancer after the lung $(3,4)$. ${ }^{131}$ I therapy is effective for most DTC patients with lung metastases, and these patients can survive for a long time after ${ }^{131}$ I treatment. Nevertheless, DTC bone metastases (BM) can cause serious complications, with the survival 


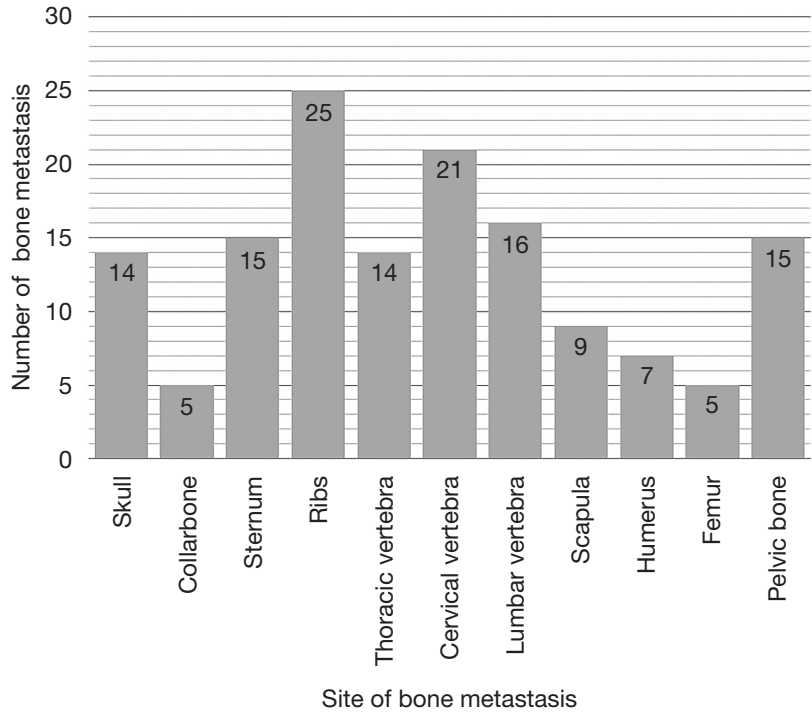

Figure 1 Site distribution of DTC bone metastatic lesions. DTC, differentiated thyroid carcinoma.

rate and the quality of life decreasing significantly (3,5-7). Moreover, there is currently no standardized therapeutic regimen for patients with DTC BM. In particular, there is controversy regarding the efficacy of ${ }^{131} \mathrm{I}$ therapy, local external beam radiotherapy (EBRT) and local surgery for $\mathrm{BM}$ lesions. In order to explore the effective treatment models for patients with DTC BM and their prognostic factors, the clinicopathological characteristics, treatment and follow-up data of all patients with DTC BM who received treatment in the Third Affiliated Hospital of Kunming Medical University between November 1, 1993 and December 31, 2016 were analyzed retrospectively.

\section{Methods}

\section{Patients studied}

This study was approved by the Ethics Committee of the Third Affiliated Hospital of Kunming Medical University, and informed consent was obtained from all the participants or their guardians before enrolment. The criteria used for the diagnosis of BM from DTC included the following: (I) previous or synchronous history of well- DTC; (II) undergoing standard surgical treatment for local and regional tumors; and (III) at least one lesion within any bone diagnosed by histological or imaging confirmation as being BM. The exclusion criteria were the following: (I) the original DTC (including primary tumor or metastatic lesion) transformed into undifferentiated thyroid carcinoma; and (II) the DTC patients did not undergo standard surgical treatment for local and regional tumors or received chemotherapy or radiotherapy or ${ }^{131} \mathrm{I}$ for any reason. Patients with inadequate thyroid-stimulating hormone (TSH) suppressive therapy were not excluded. Among the 77 patients diagnosed with DTC BM between November 1, 1993 and December 31, 2016, 60 patients met the selection criteria and enrolled, while the remaining 17 patients were excluded because of other primary cancers or improper treatment for local or regional tumors. Overall, 27 patients were male and 33 were female, with a male-to-female ratio of 1:1.2. When BM was diagnosed, 50 patients were 45 or older, 10 patients were $<45$ years old, and the median age at diagnosis of $\mathrm{BM}$ was 58 years old. The time interval from diagnosis of DTC to diagnosis of BM was 0-360 months, with a median interval of 34 months; $\mathrm{BM}$ was diagnosed simultaneously in 26 patients with primary thyroid carcinoma. The level of TSH was higher than the upper limit of the normal range in 5 cases, less than the lower limit of the normal range in 24 cases, and within the normal range in 31 cases. Twenty-nine patients with BM were from papillary thyroid carcinoma (PTC) and 31 patients were from follicular thyroid carcinoma (FTC). There were 146 $\mathrm{BM}$ in these 60 patients; solitary and multiple BM occurred in 23 cases and 37 cases, respectively, with an average of $2.43 \mathrm{BM}$ per patient. BM lesions were localized in the skull (14 cases), collarbone (5 cases), sternum (15 cases), ribs (25 cases), cervical vertebrae (14 cases), thoracic vertebrae (21 cases), lumbar vertebrae (16 cases), scapula (9 cases), humerus ( 7 cases), femur ( 5 cases) and pelvic bone (15 cases). The BM site distribution is shown in Figure 1. There were 29 cases of simple BM, and 24 cases were complicated with one organ metastasis (pulmonary); 7 cases were complicated with multiple organ metastasis ( 2 cases with lung and liver, and 4 cases with lung and brain, 1 case with liver and brain). Thirty-five cases suffered skeletal-related events (SREs), specifically, bone metastatic pain, spinal cord compression and pathological fracture occurred in 33, 7 and 3 patients, respectively.

\section{Treatment received}

Due to the advanced disease, the individualized treatment strategy is determined by the primary physician according to the patients' disease, general condition, and their expectations for life span and quality of life. Fifteen patients received only support or palliative treatment due either 
to the advanced stage of their tumor or to the presence of other medical problems. At least one of the following treatment modalities, including surgery, radiotherapy, chemotherapy or ${ }^{131} \mathrm{I}$ was given to the 45 patients with DTC BM. Surgical resection alone was performed in 5 patients, radiotherapy was performed alone in 7 patients, and ${ }^{131} \mathrm{I}$ had performed alone in 9 patients. Twenty-four patients received combined treatment: surgery combined with radiotherapy, $\mathrm{n}=2$; surgery combined with ${ }^{131} \mathrm{I}, \mathrm{n}=9$; radiotherapy combined with chemotherapy, $\mathrm{n}=5$; radiotherapy combined with ${ }^{131} \mathrm{I}, \mathrm{n}=6$; radiotherapy combined with chemotherapy and ${ }^{131} \mathrm{I}, \mathrm{n}=2$. Overall, sixteen patients underwent surgical resection for $\mathrm{BM}$ lesions; 22 patients received $\mathrm{BM}$ lesion radiotherapy (dose: 30-60 Gy, median dose: 40 Gy). Seven patients (except for BM, 3 cases with lung, and 3 cases with lung and brain, 1 case with lung and liver metastasis) received chemotherapy ( 2 cases of cisplatin + 5 -fluorouracil + doxorubicin, 5 cases of paclitaxel + cisplatin or carboplatin; the number of chemotherapy courses ranged from 1 to 6 , with a median duration of chemotherapy of 2 courses). Twenty-six patients received ${ }^{131} \mathrm{I}$ therapy (dose: 600-1,200 mCi, median dose: $800 \mathrm{mCi}$ ).

\section{Follow-up and statistical analysis}

Follow-up was completed by the patient returning to the hospital at an undefined time or by a phone call from the Medical Records Room. Up to April 2018, 34 patients died of tumor progression, and two missing patients were calculated according to death. All the data were analyzed using SPSS 22.0 software. Survival curves were generated using the Kaplan-Meier method, and the log-rank test was used for univariate analysis. For multivariate survival analysis, we used a Cox proportional hazards model, and probabilities of less than 0.05 were accepted as significantly different.

\section{Results}

\section{Univariate analysis for patients with DTC BM}

The univariate analysis showed that the prognoses for patients with simultaneous BM diagnosis were worse than those for patients diagnosed with BM after DTC treatment [5-year overall survival (OS): 27.4\% vs. $64.0 \%$, respectively, $\mathrm{P}=0.016$; Figure $2 A]$, and patients aged $\geq 45$ years old at diagnosis with $\mathrm{BM}$ had worse prognoses than did patients aged $<45$ years old (5-year OS: $29.2 \%$ vs. $72.9 \%$, respectively, $\mathrm{P}=0.031$; Figure $2 B$ ). This finding was similar to the situation of primary-DTC. We also found that the DTC $\mathrm{BM}$ patients complicated with another two or more types of organ metastases had worse prognoses than did patients complicated by less than two types of organ metastasis (5-year OS: $14.3 \%$ vs. $59.1 \%, \mathrm{P}=0.003$; Figure 2C). There were no significant differences in survival in terms of patient gender, pathological type, associated SREs or number of bone metastatic lesions (Figure 2D); nevertheless, we found that the prognoses of patients with cervical vertebra metastases were worse than those without cervical vertebra metastases (Figure 2E). It is well known that TSH levels should be kept $<0.1 \mathrm{mIU} / \mathrm{L}$ in subjects with metastasis or high-risk subjects according to the ATA 2009 and 2015 guidelines. In theory, the TSH level for DTC BM patients should be kept below that level, however, few patients in the present study are able to control this level because of poor adherence to treatments, heart diseases or other diseases. In the present study, just four patients whose TSH levels were controlled below $0.1 \mathrm{mIU} / \mathrm{L}$. Thus, patients were divided into three groups based on TSH level (< lower limit of normal, within normal, and > upper limit of normal). We found that TSH levels in patients with DTC BM were negatively related to prognosis. Patients with TSH levels not exceeding the upper limit of normal had better prognoses than did patients with TSH levels exceeding the upper limit of normal $(\mathrm{P}=0.017$; Figure $2 F)$. This suggested that TSH suppressive therapy remains very important for improving the prognosis of patients with DTC BM. The univariate analysis also indicated that patients undergoing surgical treatment for $\mathrm{BM}$ lesions $(\mathrm{P}=0.002$; Figure $3 A)$ and

${ }^{131} \mathrm{I}$ therapy had better prognoses $(\mathrm{P}=0.001$; Figure $3 B)$. In particular, patients who received ${ }^{131} \mathrm{I}$ combined with surgery had the best prognosis compared with all other treatment modes (5-year OS: $83.3 \%$ vs. $44.7 \%$, respectively, $\mathrm{P}=0.033$; Figure 3C). The univariate analysis showed that DTC BM patients receiving chemotherapy had worse prognoses than did those not receiving chemotherapy (5-year OS: $0 \%$ vs. $59.1 \%$, respectively, $\mathrm{P}=0.001$; Figure $3 D$ ). Nevertheless, there were no significant differences in survival between patients receiving or not receiving radiotherapy. The influence of various clinical characteristics and treatment methods on prognosis of 60 patients with DTC BM is shown in Table 1.

\section{Multivariate analysis for patients with DTC BM}

Seven factors were brought into the Cox regression model for multivariate analysis, including patient gender, age at diagnosis with BM, TSH level, surgical treatment for the 
A

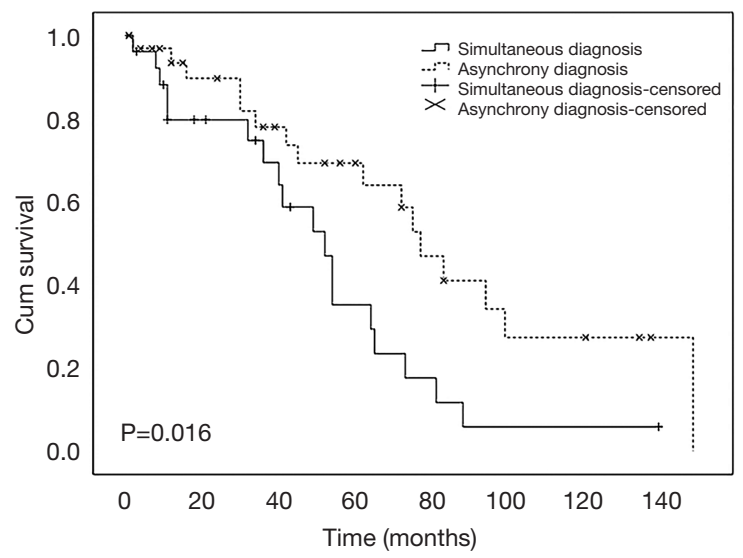

C

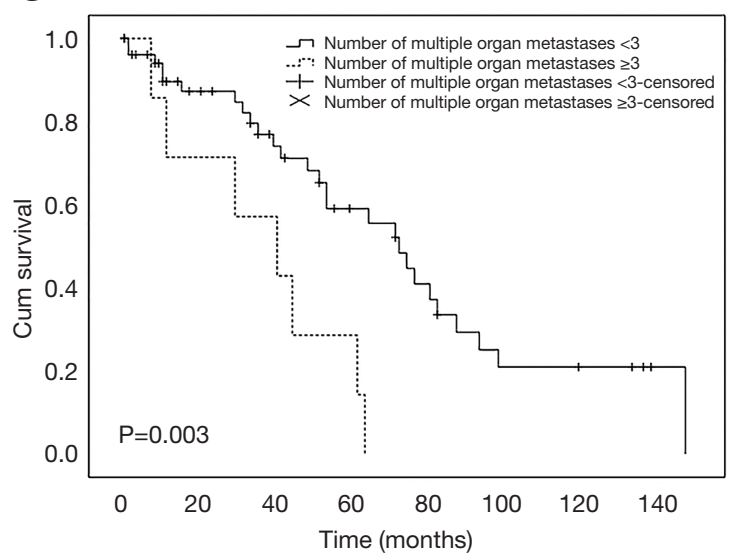

E

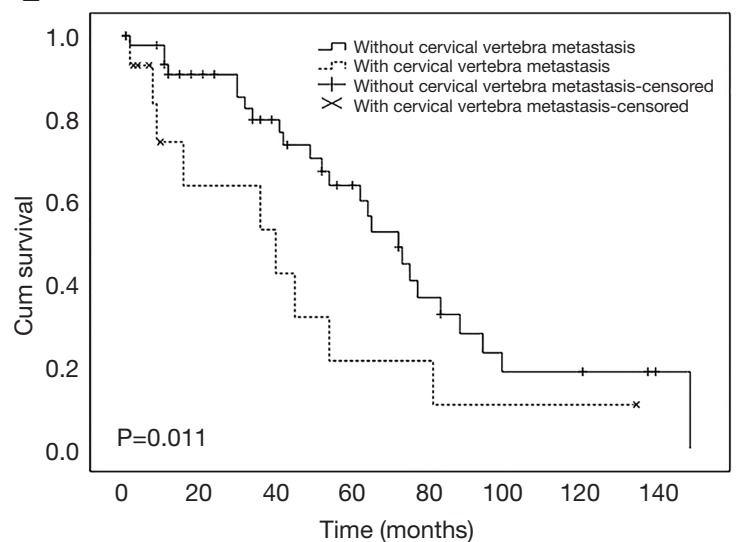

B

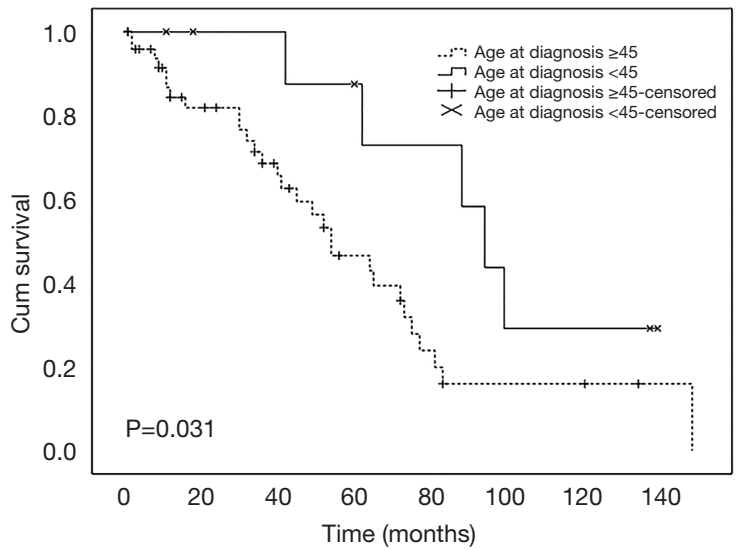

D

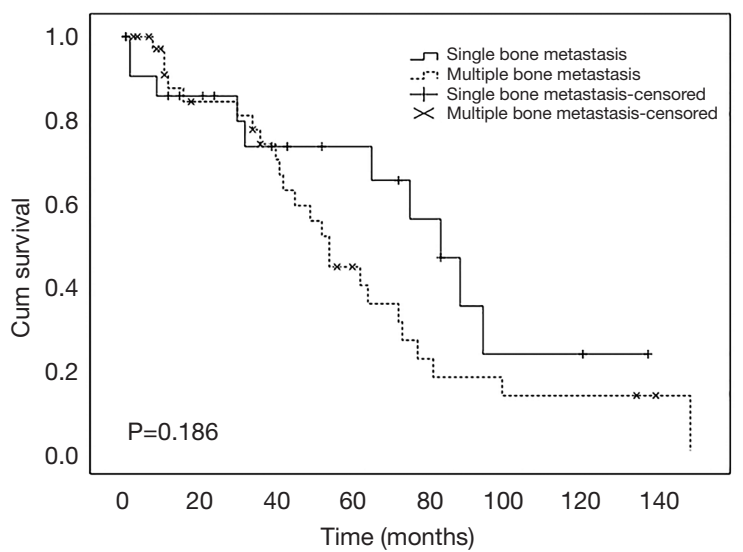

$\mathrm{F}$

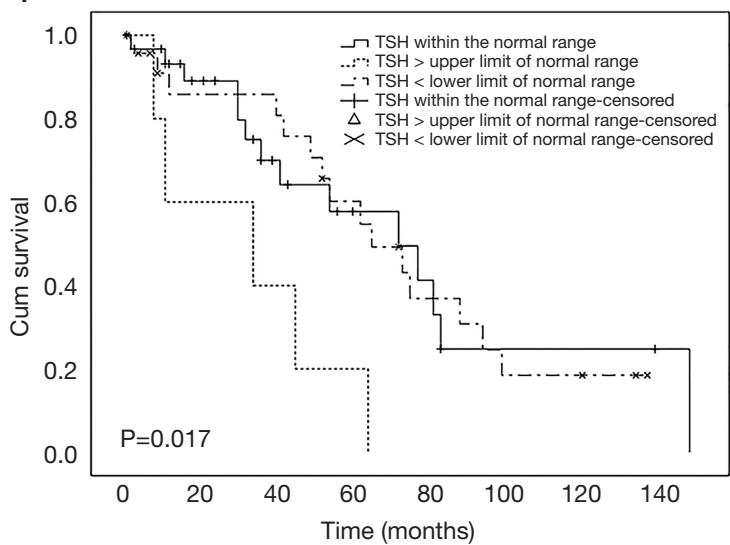

Figure 2 Effect of different clinical characteristics on survival curve in 60 patients with DTC BM. (A) Effect of time for diagnosis of BM on survival curve $(\mathrm{P}=0.016)$; ( $\mathrm{B}$ ) effect of age diagnosed as $\mathrm{BM}$ on survival curve $(\mathrm{P}=0.031)$; (C) effect of multiple organ metastasis on survival curve $(\mathrm{P}=0.003)$; (D) effect of number of $\mathrm{BM}$ on survival curve $(\mathrm{P}=0.186)$; $(\mathrm{E})$ effect of cervical spine metastasis on survival curve $(\mathrm{P}=0.011)$; (F) effect of TSH level on survival curve ( $\mathrm{P}=0.017)$. DTC, differentiated thyroid carcinoma; BM, bone metastases; TSH, thyroid-stimulating hormone. 
A

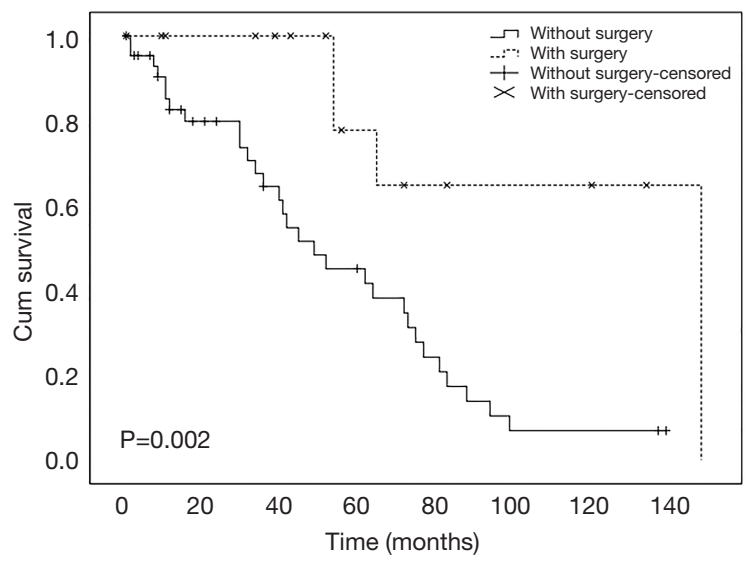

C

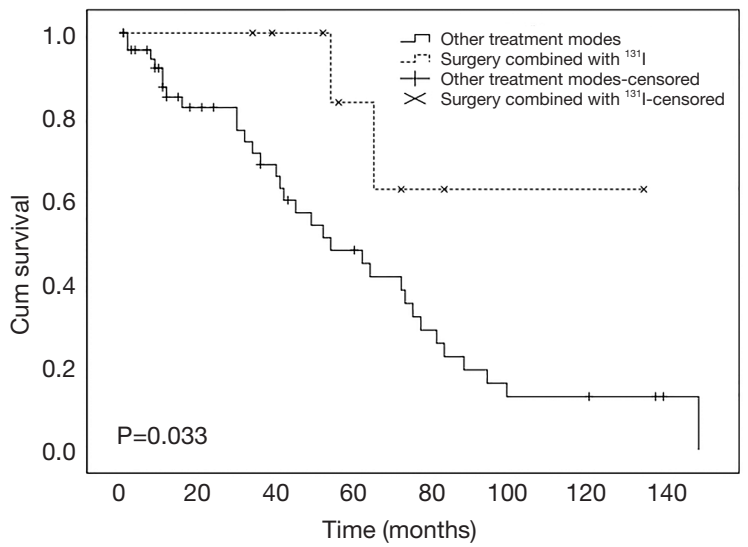

B

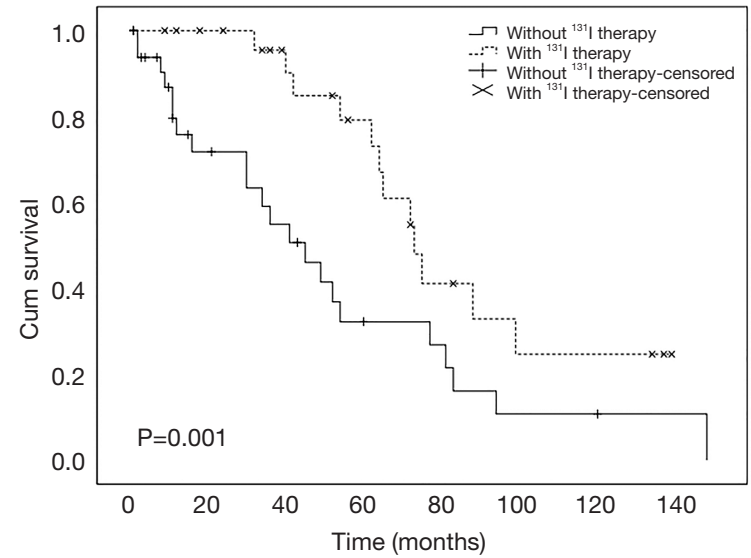

D

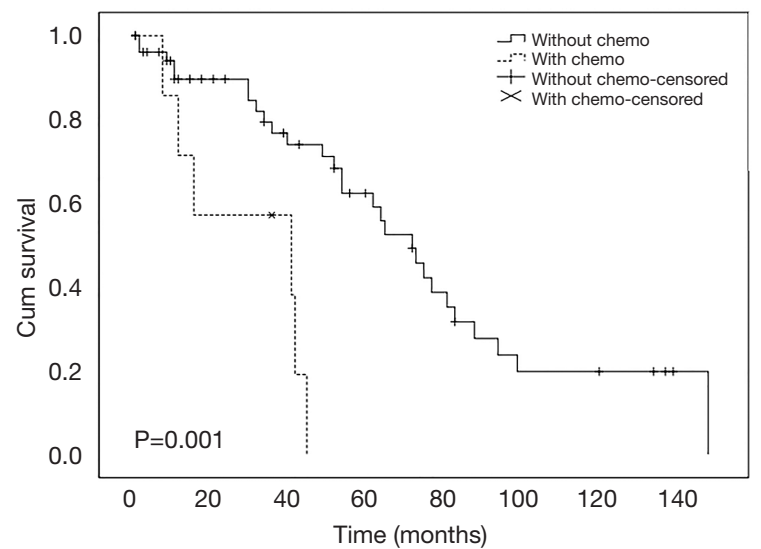

Figure 3 Effect of different treatment mode on survival curve in 60 patients with DTC BM. (A) Effect of bone metastatic lesions surgery on survival curve $(\mathrm{P}=0.002)$; (B) effect of ${ }^{131} \mathrm{I}$ therapy on survival curve $(\mathrm{P}=0.001)$; (C) effect of surgery combined with ${ }^{131} \mathrm{I}$ therapy on survival curve $(\mathrm{P}=0.033)$; ( $\mathrm{D})$ effect of chemotherapy on survival curve $(\mathrm{P}=0.001)$. DTC, differentiated thyroid carcinoma; BM, bone metastases; chemo, chemotherapy.

$\mathrm{BM}$ lesion, chemotherapy or ${ }^{131} \mathrm{I}$ therapy and presence of cervical vertebrae metastases. The results revealed that the age less than 45 years at diagnosis with BM, undergoing surgery for the BM lesion, and not receiving chemotherapy were independent factors of favorable prognosis for patients with DTC BM (Table 2).

\section{Discussion}

\section{Treatment for patients with DTC BM}

The prognosis of DTC patients is relatively good after receiving appropriate treatment, depending on the individual situation. Nevertheless, patients with DTC BM generally have worse prognoses (3,5-7). Early diagnosis and prompt and effective treatment have important influences on prognosis. Treatments for patients with DTC BM include ${ }^{131} \mathrm{I}$ therapy, surgical resection, local EBRT, systemic bisphosphonate therapy and molecular targeting therapy, as well as TSH-suppressive therapy (8-10). Even with so many therapeutic measures, the 10 -year survival rate remains low, and the efficacy of the abovementioned therapeutic measures remains controversial.

It is generally believed that the efficacy of ${ }^{131} \mathrm{I}$ in treating DTC BM depends on the iodine absorption rate of bone metastatic lesions (7). Clinical studies have shown that ${ }^{131} \mathrm{I}$ 
Table 1 Influence of different clinical characteristics and treatment methods on prognosis of 60 patients with DTC BM

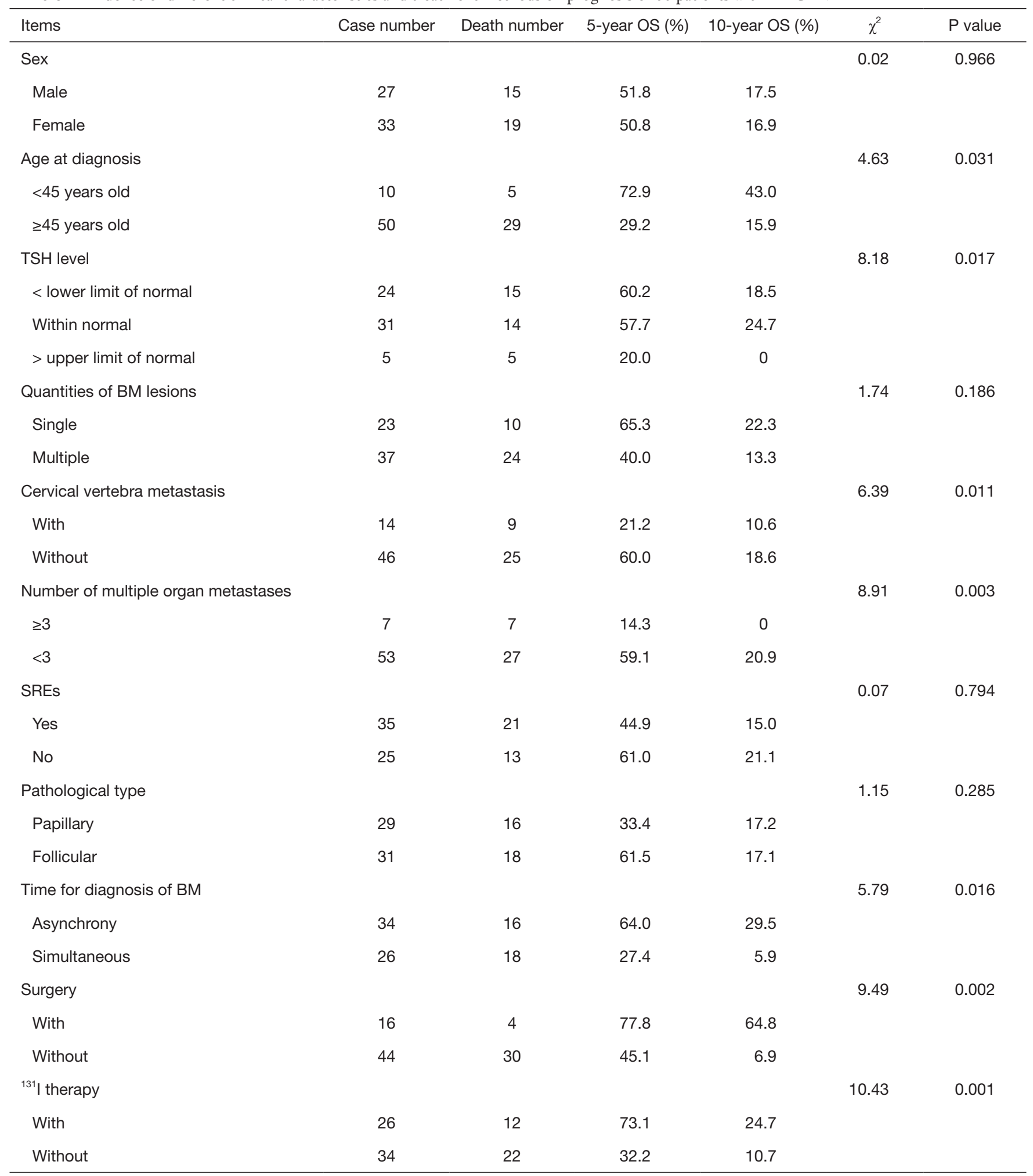

Table 1 (continued) 
Table 1 (continued)

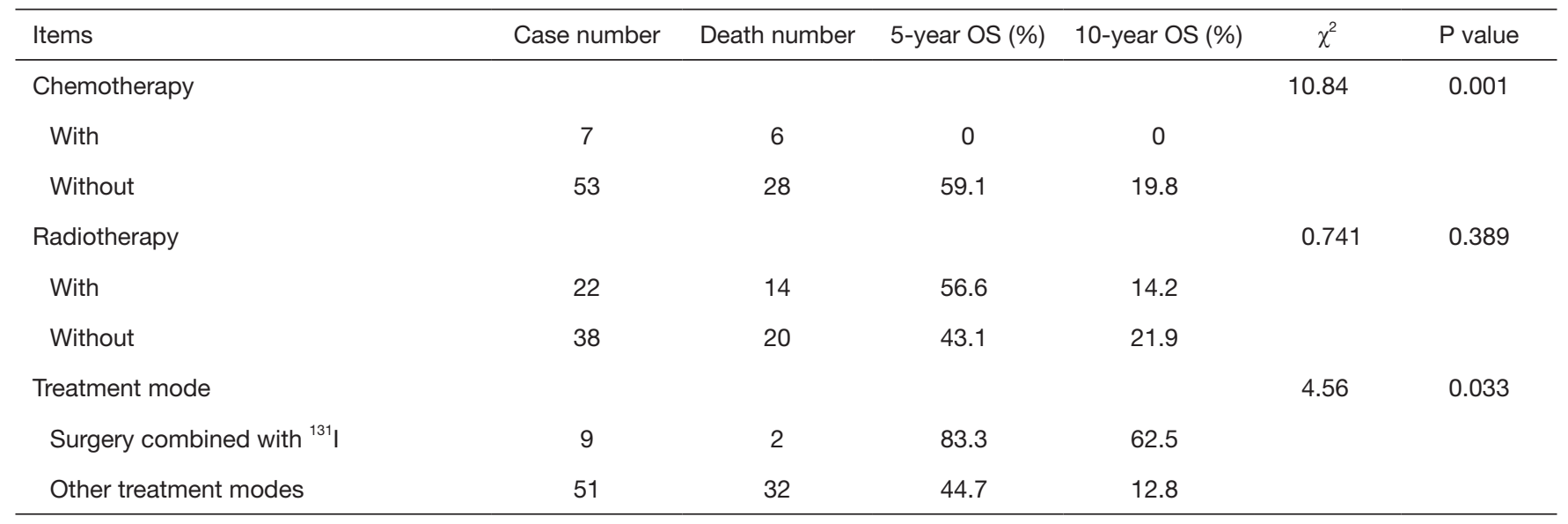

DTC, differentiated thyroid carcinoma; BM, bone metastases; OS, overall survival; TSH, thyroid-stimulating hormone; SRE, skeletal-related event.

Table 2 Multivariate analysis of Cox regression model in 60 patients with DTC BM

\begin{tabular}{|c|c|c|c|c|c|c|c|}
\hline Variables & B & SE & Wald & Sig. & $\operatorname{Exp}(B)$ & \multicolumn{2}{|c|}{$95 \% \mathrm{Cl}$ for $\operatorname{Exp}(\mathrm{B})$} \\
\hline Age $<45$ years old & -1.845 & 0.591 & 9.731 & 0.002 & 0.158 & 0.050 & 0.504 \\
\hline Chemotherapy & 1.045 & 0.514 & 4.137 & 0.042 & 2.843 & 1.039 & 7.780 \\
\hline Surgery for BM lesions & -2.308 & 0.671 & 11.842 & 0.001 & 0.099 & 0.027 & 0.370 \\
\hline
\end{tabular}

DTC, differentiated thyroid carcinoma; BM, bone metastases; B, beta; SE, standard error; Sig., significance; 95\% Cl, 95\% confidence interval.

effectively relieves bone pain, reduces serum thyroglobulin ( $\mathrm{Tg}$ ) levels, and reduces or stabilizes bone metastatic focus. Furthermore, its curative effect is better than that of routine EBRT or chemotherapy (7). A retrospective clinical study found that of 106 patients with DTC BM who received ${ }^{131}$ I treatment, 37 (34.9\%) had significantly lower serum Tg levels, and $56(52.8 \%)$ had stable serum Tg. Of the 61 patients with metastatic bone pain, 39 had a significant remission of bone pain. The overall 5- and 10-year survival rates in that study were $86.5 \%$ and $57.9 \%$, respectively (7). Petrich et al. (5) studied 107 patients with DTC BM and found that ${ }^{131} \mathrm{I}$ therapy produced long-term remission $(24 \%)$ or partial remission $(27 \%)$ in a large proportion of patients with DTC initial BM. They recommended that patients with less than three bone metastatic lesions should be treated with high-dose radioiodine with the intention of achieving complete remission. However, Schlumberger et al. (11) suggested that DTC BM are generally resistant to ${ }^{131} \mathrm{I}$ therapy. Most of the patients with BM are very old, ${ }^{131} \mathrm{I}$ uptake is poor and the effectiveness of treatment is very low (7\%); therefore, the authors thought ${ }^{131} \mathrm{I}$ should not be recommended to treat DTC BM (12). In the present study, we found that the median age for diagnosis of DTC BM was 58 years. Twenty-six patients who received ${ }^{131} \mathrm{I}$ had better 5 -year survival rates than did the 34 patients who did not receive ${ }^{131} \mathrm{I}$ therapy (nineteen patients refused indeterminate ${ }^{131}$ I treatment because of poor iodine absorption of bone metastatic lesion before treatment). This result is consistent with that of Qiu et al. (7); they found that ${ }^{131} \mathrm{I}$ therapy was an effective measure for patients with DTC BM, but it was necessary to confirm the iodine absorption of bone metastatic lesion before treatment.

Surgery is considered the preferred treatment for DTC BM (9). Bernier et al. (13) retrospectively analyzed 109 patients with DTC BM and found that the average survival time was 6.2 years for patients undergoing complete resection 
of bone metastatic lesions ( 24 patients), 4.2 years for patients undergoing partial excision (60 patients) and 2.5 years for the patients not undergoing any surgical treatment, respectively $(\mathrm{P}<0.05)$. Our study found that surgical resection of BM lesions was beneficial for prognosis, and multivariate analysis also showed that surgery was an independent prognostic factor; the hazard ratio as to whether or not to perform surgery was 0.099 (range, $0.027-0.370$ ), suggesting that surgery significantly reduced the death rate up to 10.1 (2.7-37.0) times. Surgical management of bone metastatic lesions not only removes metastatic lesions but also relieves nerve compression and local pain. Nevertheless, Quan et al. (14) suggested that surgery should be performed only on patients with spinal cord compression, intractable pain with neurological symptoms or cervical instability. They also stressed that surgery might produce surgical complications and may affect daily activities in DTC patients with cervical vertebra metastases. Therefore, it is necessary to take full account of the effectiveness and adverse consequences of surgery when planning surgical intervention for patients with DTC BM. The present study also found that, for patients with DTC BM, surgical treatment of BM combined with ${ }^{131} \mathrm{I}$ therapy achieved better results than any other treatment modalities. Surgical treatment of BM combined with ${ }^{131} \mathrm{I}$ therapy was shown to be a priority treatment for DTC BM in the present study.

EBRT may be a good choice when ${ }^{131} \mathrm{I}$ is ineffective; it can control disease progression rapidly and reduce metastatic lesion pain (15). Kotecha et al. (16) reported that gamma knife therapy was an effective tool for skull or base metastasis of DTC. Proye et al. (17) retrospectively analyzed 28 cases of DTC BM treated with EBRT and found that 23 patients $(82 \%)$ with metastatic tumors were controlled well. However, our study found that patients who received EBRT had better prognoses than did those not receive EBRT, although the difference was not significant. Similarly, Bernier et al. (13) compared the prognoses of patients receiving radiotherapy (39 patients) with those of patients not receiving radiotherapy ( 70 patients) and found that the difference of median survival times was not significant between the two groups ( 4.3 vs. 3.8 years, $\mathrm{P}>0.05$ ). Frassica et al. (18) stated that the objective of EBRT was to alleviate pain and neurological complications of bone lesions but not to improve prognosis. More accurate and reliable data on the efficacy of EBRT in the management of DTC BM are lacking; however, it is believed that $70 \%$ of patients experience pain relief with palliative EBRT (18).
Therefore, whether EBRT is effective at improving the OS for patients with DTC BM remains to be confirmed by further prospective clinical studies.

Vertebrae are the most common metastatic sites for patients with DTC BM (19), occurring in $34.9 \%$ in the present study. We found that prognosis for patients with cervical vertebral metastases was worse than for those without cervical vertebral metastases. This finding may be due to the unique anatomical structure and junction of the cervical vertebrae, making the operation very difficult (20). Moreover, it is not known whether the higher position of the cervical spine metastasis reduces the quality of life of the patients. Univariate analysis in this study showed that the prognoses of patients with DTC BM were further aggravated by chemotherapy, and multivariate analysis showed that chemotherapy was a poor independent risk factor, suggesting that DTC BM is insensitive to chemotherapy and that chemotherapy introduces severe side-effects, as in primary DTC disease. Similarly, no similar study proved that chemotherapy improved the prognoses for patients with DTC BM $(21,22)$. Univariate analysis in the present study also showed that controlling TSH could significantly improve prognosis in patients with DTC BM $(\mathrm{P}=0.017)$; this is consistent with the results form Hovens (23) and Orita et al. (24). Additionally, in the present study, few patients received ideal effect of TSH suppressive therapy, we are not sure whether it is one of reasons which caused BM in these patients. These results suggest that thyroid functions should be monitored regularly in these patients.

In addition to the treatment described above, Orita et al. $(24,25)$ retrospectively studied 22 patients with DTC BM who underwent zoledronic acid therapy and performed a subsequent prospective study suggesting that zoledronic acid was a safe, well-tolerated and effective treatment for palliation in DTC BM patients. No patients were treated with zoledronic acid in the present study; therefore, we cannot evaluate its efficacy. Radiofrequency ablation (26) reduced pain from thyroid cancer with BM, and cryotherapy (27) is another technique for the treatment of BM from thyroid cancer; nevertheless, their effectiveness is controversial and requires further study.

\section{Other factors influencing the prognosis of DTC BM}

Age of onset is one of the most important factors affecting prognosis in DTC patients. This result was 
the same for DTC patients with distant metastasis (28). The univariate analysis in the present study showed that prognosis for patients $<45$ years of age at diagnosis of DTC $\mathrm{BM}$ was significantly better than that for patients $\geq 45$ years of age; the 5-year survival rates of the two groups were $72.9 \%$ and $29.2 \%$, respectively, and the 10 -year survival rates were $43.0 \%$ and $15.9 \%$, respectively. Multivariate analysis also showed that age was an independent prognostic factor. It has been reported in some studies that the number of BM lesions influenced prognosis $(29,30)$. However, our results and the study of Do et al. (31) did not confirm this finding. The follow-up data in the present study showed that DTC BM often results in severe SREs, including pain, spinal cord compression, pathological fractures and hypercalcemia. Pittas et al. (32) reported that SREs did not affect survival rates, consistent with the results of the present study.

As everyone knows, a prospective clinical trial in which subjects are matched with similar tumor characteristics and randomized to receive EBRT, surgery, ${ }^{131} \mathrm{I}$, chemotherapy or combinations, which helps to avoid grouping bias and leads to an accurate conclusion. However, because of the very low incidence and a relatively long expectation of survival for the patients with DTC BM, it is difficult to accumulate a large sample with a long-term follow-up. We recognize that there are several limitations with our retrospective study, including incomplete documentation and problematic verification of information. Also, the number of the patients was not sufficiently large and some patients received inappropriate chemotherapy. Despite these limitations, we found that the age at diagnosis of BM and TSH level significantly affected prognosis in patients with DTC BM, proving again from the opposite side that DTC remains insensitive to chemotherapy, even invasive DTC cells metastasize to bone and other organs. It may be useful for making reasonable therapeutic decisions when clinicians are faced with choosing the treatment model.

\section{Conclusions}

In conclusion, this study suggested that once BM occur in patients with DTC, the treatment becomes more difficult and the prognosis deteriorates significantly. We found that age at diagnosis with BM less than 45 years, surgical resection of bone metastatic lesions and not receiving chemotherapy were independent factors of favorable prognosis for patients with DTC BM. Surgery combined with ${ }^{131} \mathrm{I}$ is preferred, depending on individual conditions, although the efficacy of these treatments is controversial. The effectiveness of EBRT for DTC BM requires further studies.

\section{Acknowledgments}

Funding: This work was funded by grants from the National Natural Science Foundation of China (81560470 and 81773127), Special Foundation of Young and Middle-aged Academic Leaders Reserve Talent in Yunnan Province (2015HB086), Special Foundation of Yunnan Medical leaders (L-2017025), and Yunnan Province Basic Research Program (2018FE001-058/246).

\section{Footnote}

Conflicts of Interest: All authors have completed the ICMJE uniform disclosure form (available at http://dx.doi. org/10.21037/tcr.2019.12.43). The authors have no conflicts of interest to declare.

Ethical Statement: The authors are accountable for all aspects of the work in ensuring that questions related to the accuracy or integrity of any part of the work are appropriately investigated and resolved. The study was conducted in accordance with the Declaration of Helsinki (as revised in 2013). This study was approved by the Ethics Committee of the Third Affiliated Hospital of Kunming Medical University (No. 2018-0153), and informed consent was obtained from all the participants or their guardians before enrolment.

Open Access Statement: This is an Open Access article distributed in accordance with the Creative Commons Attribution-NonCommercial-NoDerivs 4.0 International License (CC BY-NC-ND 4.0), which permits the noncommercial replication and distribution of the article with the strict proviso that no changes or edits are made and the original work is properly cited (including links to both the formal publication through the relevant DOI and the license). See: https://creativecommons.org/licenses/by-nc-nd/4.0/.

\section{References}

1. Jasim S, Ozsari L, Habra MA. Multikinase inhibitors use in differentiated thyroid carcinoma. Biologics 2014;8:281-91.

2. Xing $M$, Haugen BR, Schlumberger M. Progress in 
molecular-based management of differentiated thyroid cancer. Lancet 2013;381:1058-69.

3. Zanotti-Fregonara P, Rubello D, Hindié E. Bone metastases of differentiated thyroid cancer: the importance of early diagnosis and 131I therapy on prognosis. J Nucl Med 2008;49:1902-3.

4. Orita $Y$, Sugitani I, Matsuura M, et al. Prognostic factors and the therapeutic strategy for patients with bone metastasis from differentiated thyroid carcinoma. Surgery 2010;147:424-31.

5. Petrich T, Widjaja A, Musholt TJ, et al. Outcome after radioiodine therapy in 107 patients with differentiated thyroid carcinoma and initial bone metastases: side-effects and influence of age. Eur J Nucl Med 2001;28:203-8.

6. Mazziotti G, Formenti AM, Panarotto MB, et al. Real-life management and outcome of thyroid carcinoma-related bone metastases: results from a nationwide multicenter experience. Endocrine 2018;59:90-101.

7. Qiu ZL, Song HJ, Xu YH, et al. Efficacy and survival analysis of 131I therapy for bone metastases from differentiated thyroid cancer. J Clin Endocrinol Metab 2011;96:3078-86

8. Lin JD, Lin SF, Chen ST, et al. Long-term follow-up of papillary and follicular thyroid carcinomas with bone metastasis. PLoS One 2017;12:e173354.

9. Haugen BR, Alexander EK, Bible KC, et al. 2015 American Thyroid Association Management Guidelines for Adult Patients with Thyroid Nodules and Differentiated Thyroid Cancer: The American Thyroid Association Guidelines Task Force on Thyroid Nodules and Differentiated Thyroid Cancer. Thyroid 2016;26:1-133.

10. Wexler JA. Approach to the thyroid cancer patient with bone metastases. J Clin Endocrinol Metab 2011;96:2296-307.

11. Schlumberger $M$, Challeton C, De Vathaire F, et al. Radioactive iodine treatment and external radiotherapy for lung and bone metastases from thyroid carcinoma. J Nucl Med 1996;37:598-605.

12. Durante C, Haddy N, Baudin E, et al. Long-term outcome of 444 patients with distant metastases from papillary and follicular thyroid carcinoma: benefits and limits of radioiodine therapy. J Clin Endocrinol Metab 2006;91:2892-9.

13. Bernier MO, Leenhardt L, Hoang C, et al. Survival and therapeutic modalities in patients with bone metastases of differentiated thyroid carcinomas. J Clin Endocrinol Metab 2001;86:1568-73.

14. Quan GM, Pointillart V, Palussière J, et al.
Multidisciplinary treatment and survival of patients with vertebral metastases from thyroid carcinoma. Thyroid 2012;22:125-30.

15. Dadu R, Cabanillas ME. Optimizing therapy for radioactive iodine-refractory differentiated thyroid cancer: current state of the art and future directions. Minerva Endocrinol 2012;37:335-56.

16. Kotecha R, Angelov L, Barnett GH, et al. Calvarial and skull base metastases: expanding the clinical utility of Gamma Knife surgery. J Neurosurg 2014;12 1 Suppl:91-101.

17. Proye CA, Dromer DH, Carnaille BM, et al. Is it still worthwhile to treat bone metastases from differentiated thyroid carcinoma with radioactive iodine? World J Surg 1992;16:640-5; discussion 645-6.

18. Frassica DA. General principles of external beam radiation therapy for skeletal metastases. Clin Orthop Relat Res 2003;(415 Suppl):S158-64.

19. Osorio M, Moubayed SP, Su H, et al. Systematic review of site distribution of bone metastases in differentiated thyroid cancer. Head Neck 2017;39:812-8.

20. Mesfin A, Buchowski JM, Gokaslan ZL, et al. Management of metastatic cervical spine tumors. J Am Acad Orthop Surg 2015;23:38-46.

21. Muresan MM, Olivier P, Leclère J, et al. Bone metastases from differentiated thyroid carcinoma. Endocr Relat Cancer 2008;15:37-49.

22. Smallridge RC, Ain KB, Asa SL, et al. American Thyroid Association guidelines for management of patients with anaplastic thyroid cancer. Thyroid 2012;22:1104-39.

23. Hovens GC, Stokkel MP, Kievit J, et al. Associations of serum thyrotropin concentrations with recurrence and death in differentiated thyroid cancer. J Clin Endocrinol Metab 2007;92:2610-5.

24. Orita Y, Sugitani I, Toda K, et al. Zoledronic acid in the treatment of bone metastases from differentiated thyroid carcinoma. Thyroid 2011;21:31-5.

25. Orita Y, Sugitani I, Takao S, et al. Prospective evaluation of zoledronic acid in the treatment of bone metastases from differentiated thyroid carcinoma. Ann Surg Oncol 2015;22:4008-13.

26. Toyota N, Naito A, Kakizawa H, et al. Radiofrequency ablation therapy combined with cementoplasty for painful bone metastases: initial experience. Cardiovasc Intervent Radiol 2005;28:578-83.

27. Robinson D, Yassin M, Nevo Z. Cryotherapy of musculoskeletal tumors--from basic science to clinical results. Technol Cancer Res Treat 2004;3:371-5. 
28. Shoup M, Stojadinovic A, Nissan A, et al. Prognostic indicators of outcomes in patients with distant metastases from differentiated thyroid carcinoma. J Am Coll Surg 2003;197:191-7.

29. Tokuhashi Y, Matsuzaki H, Toriyama S, et al. Scoring system for the preoperative evaluation of metastatic spine tumor prognosis. Spine (Phila Pa 1976) 1990;15:1110-3.

30. Tomita K, Kawahara N, Kobayashi T, et al. Surgical strategy for spinal metastases. Spine (Phila Pa 1976)

Cite this article as: Wang S, Luo J, Zhong Z, Qi X, Liu T, Qin R, Zhao Z, Xi Y, Sun R, Wu Y, Sun C. Treatment and prognosis for patients with differentiated thyroid carcinoma bone metastases. Transl Cancer Res 2020;9(2):972-982. doi: $10.21037 /$ tcr.2019.12.43
2001;26:298-306.

31. Do MY, Rhee Y, Kim DJ, et al. Clinical features of bone metastases resulting from thyroid cancer: a review of 28 patients over a 20-year period. Endocr J 2005;52:701-7.

32. Pittas AG, Adler M, Fazzari M, et al. Bone metastases from thyroid carcinoma: clinical characteristics and prognostic variables in one hundred forty-six patients. Thyroid 2000;10:261-8. 